\title{
THE VIBRATIONAL STABILITY OF WHITE DWARFS
}

\author{
P. J. Ledoux* and E. Sauvenier-Goffin \\ Institut d'Astrophysique, Université de Liége \\ Received December 31, 1949
}

\section{ABSTRACT}

The condition of vibrational stability of white dwarf stars is established and studied in detail for the two main nuclear reactions which have been considered in connection with the generation of energy in these stars (carbon cycle and proton-proton reaction) when it takes place in the central region or in an external layer. It is shown that nuclear reactions would render the white dwarfs unstable except in a very special case, and hence it seems that the generation of energy in these stars must definitely be attributed to some other cause.

\section{INTRODUCTION}

The white dwarf stars constitute probably the most numerous class of stellar objects next to the main sequence. However, until now, they have been studied mainly from the point of view of pure hydrostatic equilibrium only, and it is useful to extend to them the other criteria of internal structure provided by the conditions of stability.

Some time ago A. B. Severny ${ }^{1}$ touched upon the question of their dynamical stability but only in a formal way. Since, in this respect, the value $\gamma=\frac{4}{3}$ of the ratio of specific heats is critical for gaseous stars, while in a white dwarf, in which relativistic degeneracy develops, the value of $\Gamma_{P}$ in the adiabatic relation $\delta P / P=\Gamma_{P}(\delta \rho / \rho)$ tends toward $\frac{4}{3}$, he concluded that the limit of dynamical stability would be approached at the same time as a state of complete relativistic degeneracy.

In a preceding paper" which will be cited as "Paper I" in the following, one of us has studied the dynamical stability of white dwarfs, starting from the exact equation of state. If a star is stable toward convection, it can be shown ${ }^{3}$ that purely radial perturbations are the most dangerous for its dynamical stability, and hence, in this case, the study has been restricted to the fundamental mode of radial pulsation.

It was found that, instead of decreasing, the dynamical stability actually increases with the extent of the relativistic degenerate region. This can be understood easily on the basis of the first approximation for the frequency of pulsation $\sigma$ given in that paper, namely,

$$
\sigma^{2}=-\frac{\left(3 \bar{\Gamma}_{P}-4\right) \Omega}{I}
$$

the ratio of the moment of inertia $I$ to the gravitational potential $\Omega$ tending toward zero more rapidly than $\left(3 \bar{\Gamma}_{P}-4\right)$, when the whole configuration approaches a state of complete relativistic degeneracy. These degenerate configurations are dynamically stable in all cases, and the limiting mass keeps a purely hydrostatic meaning. We are not concerned here with the possible effects of extra forces, such as centrifugal forces in rapidly rotating configurations or the effects of rotational instability, ${ }^{4}$ and we shall limit our-

* Associé du Fonds National de la Recherche Scientifique.

${ }^{1}$ C.R. Acad. Sci., U.R.S.S., 31, No. 2, 109, 1941.

2 E. Sauvenier-Goffin, Ann. d'ap., 12, 39, 1949.

${ }^{3}$ P. Ledoux, Mém. Soc. R. Sci. Liêge, 4ème sér., Vol. 9, No. 1, chap. iii, 1949.

${ }^{4}$ F. Hoyle, M.N., 107, 231, 1947. 
selves, as in the previous paper, to spherical configurations with negligible rotation or none at all.

In this paper we wish to discuss the condition of vibrational stability, which seems particularly interesting because it involves the generation of energy in white dwarfs, a question which has been much debated recently. If it is attributed to nuclear reactions, many difficulties arise from the point of view of instantaneous hydrostatic equilibrium, ${ }^{5}$ as well as from the point of view of past and future evolution. ${ }^{6}$ However, in these aspects of the problem it is always possible to find a way out of the difficulties by adopting special abundances or a particular stratification of the elements. As far as vibrational stability is concerned, it is the form of the law of generation of energy which matters, and this cannot be varied at will.

\section{FUNDAMENTAL EQUATIONS}

We shall generally use Chandrasekhar's notations, ${ }^{7}$ in which the mean electronic concentration, $n$, is expressed in terms of the temperature, $T$, and the variable $x=p_{0} / m c$, by

where

$$
n=a x^{3}\left[1+\pi^{2}\left(\frac{k T}{m c^{2}}\right)^{2} \frac{2 x^{2}+1}{2 x^{4}}+\ldots\right]
$$

$$
a=\frac{8 \pi m^{3} c^{3}}{3 h^{3}}
$$

The density $\rho$ is related to $n$ and the mean molecular weight per electron, $\mu_{e}$, by

$$
\rho=n \mu_{e} m_{H},
$$

where $m_{H}$ is the mass of the proton.

The pressure $P$ can be written as

if

$$
P=A f(x)\left[1+4 \pi^{2}\left(\frac{k T}{m c^{2}}\right)^{2} \frac{x\left(x^{2}+1\right)^{1 / 2}}{f(x)}+\ldots\right]
$$

$$
A=\frac{\pi m^{4} c^{5}}{3 h^{3}} \quad \text { and } \quad f(x)=x\left(2 x^{2}-3\right)\left(x^{2}+1\right)^{1 / 2}+3 \sin h^{-1} x .
$$

In terms of the same variable, the internal energy $E$ per unit volume is given by

where

$$
E=A g(x)\left[1+4 \pi^{2}\left(\frac{k T}{m c^{2}}\right)^{2} \frac{\left(3 x^{2}+1\right)\left(x^{2}+1\right)^{1 / 2}-\left(2 x^{2}+1\right)}{x g(x)}+\ldots\right],
$$

$$
g(x)=8 x^{3}\left[\left(x^{2}+1\right)^{1 / 2}-1\right]-f(x) .
$$

In all these expressions, the terms in $T^{2}$ are very small and are usually neglected.

If we denote by $\delta r$, the displacement of a small element of matter in the course of a perturbation and by primes the corresponding Eulerian variations, the equation of continuity can be written in the form

$$
\rho^{\prime}=-\rho \operatorname{div} \delta r-\delta r \cdot \operatorname{grad} \rho ;
$$

${ }^{5}$ R. E. Marshak, Ap. J., 92, 321, 1940.

${ }^{6}$ A. Eddington, M.N., 99, 575, 1939; Hoyle, op. cit., p. 253.

${ }^{7}$ S. Chandrasekhar, An Introduction to the Study of Stellar Structure (Chicago: University of Chicago Press, 1939), chaps. $x$ and $x i$. 
and the equation of conservation of energy per unit volume is

$$
E^{\prime}+(E+P) \operatorname{div} \delta r+\delta r \cdot \operatorname{grad} E=-\frac{i}{\sigma}\left[(\rho \epsilon)^{\prime}-\operatorname{div} F^{\prime}\right]
$$

where $\epsilon$ is the rate of generation of energy per unit mass and $F$ is the total flux due to radiation and conduction.

For adiabatic pulsations the right-hand member of equation (9) cancels out, and it can easily be seen from Paper I and the preceding definitions that, neglecting small terms in $T^{2}$, the left-hand member expressed in terms of $P^{\prime}$ and $\rho^{\prime}$ reduces to

where

$$
P^{\prime}=\Gamma_{P} \frac{P}{\rho} \rho^{\prime}
$$

$$
\Gamma_{P}=\frac{8 x^{5}}{3\left(x^{2}+1\right)^{1 / 2} f(x)}
$$

This corresponds to the fact that, to the same degree of approximation, matter inside a white dwarf can be considered as autobarotropic.

In that case it was shown in Paper I that the differential equation for adiabatic radial pulsations in terms of $\xi=\delta r / r$, can be written

$$
F(\xi) \equiv \frac{d}{d r}\left(\Gamma_{P} P r^{4} \frac{d \xi}{d r}\right)+\xi\left\{\sigma^{2} \rho r^{4}+r^{3} \frac{d}{d r}\left[\left(3 \Gamma_{P}-4\right) P\right]\right\}=0,
$$

in perfect analogy to the case of gaseous stars, ${ }^{8}$ which immediately justifies formula (1) for the frequency of the fundamental mode.

As $\Gamma_{P}$ tends toward $\frac{5}{3}$ when $x$ tends toward zero, equation (12) goes over continuously into the ordinary equation of radial pulsation for a monatomic gas, as it should, when we reach the nondegenerate fringe of the star. The solutions of equation (12), satisfying the boundary conditions,

$$
\begin{aligned}
& \delta r=0 \quad \text { at } \quad r=0, \\
& P^{\prime}+\delta r \cdot \operatorname{grad} P=0 \quad \text { at } \quad r=R,
\end{aligned}
$$

form a complete set of orthogonal functions with respect to $\rho r^{4} \delta r$.

For nonadiabatic pulsations we must use the complete equation of conservation of energy, and an extra term appears in equation (12), which becomes ${ }^{9}$

where

$$
F(\xi)=-\frac{i r^{3}}{\sigma} \frac{d}{d r}\left\{\left(\Gamma_{T}-1\right)\left[(\rho \epsilon)^{\prime}-\operatorname{div} F^{\prime}\right]\right\}
$$

$$
\Gamma_{T}=\frac{4 x^{2}+5}{3\left(x^{2}+1\right)}
$$

Physically, $\Gamma_{T}$ relates the variations of temperature $T^{\prime}$ to the variations of density $\rho^{\prime}$ in the case of an adiabatic modification. Indeed, if, in equation (8) and in the left-hand member of equation (9) equaled to zero, we express $\rho^{\prime}$ and $E^{\prime}$ in terms of $x^{\prime}$ and $T^{\prime}$ and replace $E$ and $P$ by their values in equations (4) and (6), taking the terms in $T^{2}$ into consideration, we obtain

$$
T^{\prime}=\left(\Gamma_{T}-1\right) \frac{T}{\rho} \rho^{\prime}+\delta r\left[\left(\Gamma_{T}-1\right) \frac{T}{\rho} \operatorname{grad} \rho-\operatorname{grad} T\right] .
$$

${ }^{8}$ P. Ledoux and C. L. Pekeris, $A$ p. $J$., 94, 124, 1941.

${ }^{9}$ S. Rosseland, The Pulsation Theory of Variable Stars (Oxford: Clarenden Press, 1949), chap. ii. 
Again $\Gamma_{T}$ tends to $\frac{5}{3}$ when $x$ tends to zero, so that the right-hand member of equation (14) also goes over continuously into the corresponding expression for monatomic gas, when we reach the gaseous envelope of the white dwarfs.

Using the orthogonality properties of the solutions of equation (12) and the perturbation method, ${ }^{10}$ one finds, for the perturbation $\sigma^{\prime}$ of $\sigma$ due to the second member of equation (14), the following expression:

$$
\sigma^{\prime}=\frac{1}{2 i \sigma^{2}} \int_{0}^{R} \frac{d}{d r}\left\{\left(\Gamma_{T}-1\right)\left[(\rho \epsilon)^{\prime}-\operatorname{div} F^{\prime}\right]\right\} r^{3} \xi d r
$$

Hence the condition of vibrational stability is

$$
\int_{0}^{R} \frac{d}{d r}\left\{\left(\Gamma_{T}-1\right)\left[(\rho \epsilon)^{\prime}-\operatorname{div} F^{\prime}\right] r^{3} \xi d r<0 .\right.
$$

It is somewhat more practical to express this condition in Lagrangian co-ordinates, using $m(r)$ as an independent variable. After an integration by parts and denoting variations following the motion by $\delta$, we have

$$
\delta f=f^{\prime}+\delta r \frac{d f}{d r},
$$

and the condition of vibrational stability becomes

$$
\int_{0}^{M}\left(\Gamma_{T}-1\right) \frac{\delta \rho}{\rho} \delta\left[\epsilon-\frac{d\left(4 \pi r^{2} F\right)}{d m}\right] d m<0,
$$

which is the form in which this condition will be applied in the following paragraph.

\section{EXPLICIT EXPRESSIONS OF THE DIFFERENT QUANTITIES IN EQUATION (18) FOR DEGENERATE AND NONDEGENERATE MATTER}

For the sake of simplicity we shall suppose that a white dwarf can be considered as composed of a large, completely degenerate core of mass $M_{d}$ and a gaseous envelope.

We have to express $\delta \epsilon, \delta\left[d\left(4 \pi r^{2} F\right) / d m\right]$, etc., explicitly for degenerate and nondegenerate matter. The necessary algebra is much simplified by the property of $\xi$ discovered in Paper I, where it was shown that, for all practical purposes, $\xi$ can be considered a constant in a typical white dwarf. The special behavior of $\xi$, illustrated in Figure 1, results from the small central condensation of these configurations and the regular increase of $\Gamma_{P}$ from the center toward the surface. come

Treating $\xi$ as a constant, equations (8) and (16), using the transformation (17), be-

$$
\begin{aligned}
& \frac{\delta \rho}{\rho}=-3 \xi \\
& \frac{\delta T}{T}=\left(\Gamma_{T}-1\right) \frac{\delta \rho}{\rho}=-\left(\Gamma_{T}-1\right) 3 \xi .
\end{aligned}
$$

For nuclear reactions, $\epsilon$ can be written as

and its variation is given by

$$
\epsilon=\epsilon_{0} \rho T^{\nu}
$$

$$
\frac{\delta \epsilon}{\epsilon}=\frac{\delta \rho}{\rho}+\nu \frac{\delta T}{T}=-3 \xi\left[1+\nu\left(\Gamma_{T}-1\right)\right],
$$

${ }^{10}$ Rosseland, op. cit., chap. iv, $\S 2$. 
where $\Gamma_{T}$ is given by equation (15) in the degenerate part and is equal to $\frac{5}{3}$ in the gaseous envelope.

The radiative flux, $F_{R}$, is given by

$$
F_{R}=-\frac{4 \pi c r^{2}}{\kappa_{R}} \frac{d p_{R}}{d m}
$$

where $p_{R}=\frac{1}{3} a T^{4}$ is the pressure of radiation.

But in the degenerate core the flux due to the electronic conductivity, $\lambda$, becomes very important, and we can define a coefficient of conductive opacity $\kappa_{C}=4 a c T^{3} / 3 \rho \lambda$ such that the total flux becomes

$$
F=F_{R}+F_{C}=-\frac{4 \pi r^{2} c}{\kappa} \frac{d p_{R}}{d m}
$$

where the total opacity, $\kappa$, is defined by $\kappa^{-1}=\kappa_{C}^{-1}+\kappa_{R}^{-1}$. Because of the different roles played by the conductivity in the core and the envelope, it seems best to compute the perturbation of $F$ separately for each region.

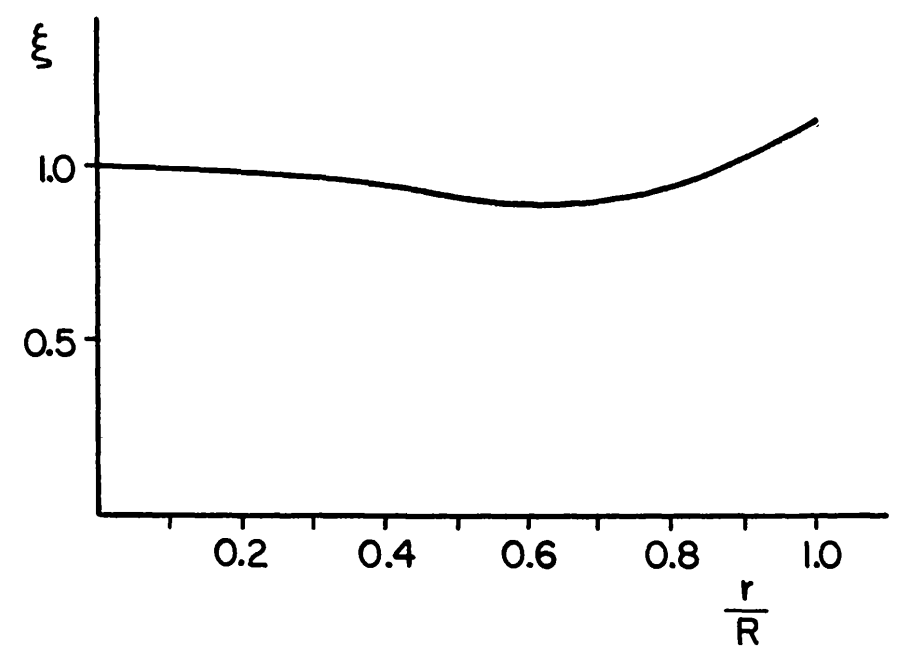

FIG. 1.-The variation in the amplitude of adiabatic pulsation of white dwarf star

a) DEGENERATE CORE

For this region the expressions given by Kothari ${ }^{11}$ are quite sufficient for our purpose, and we can write them

and

$$
\kappa_{R}=a T^{-2} \quad \text { and } \quad \kappa_{C}=\frac{a}{\beta} \frac{T^{2}}{\rho^{2}} \ln Q
$$

where

$$
F_{C}=\beta F_{R} \frac{\rho^{2} T^{-4}}{\ln Q}
$$

$$
Q=1+\vartheta \rho^{2 / 3},
$$

$a, \beta$, and $\vartheta$ being constants whose numerical values do not interest us at present.

From equation (22), using equations (19), (20), and (24) and the definition of $p_{R}$, we obtain, after some transformations,

${ }^{11} M . N ., 13,61,1932$.

$$
\frac{\delta F_{R}}{F_{R}}=2 \xi\left[\frac{T x}{\left(x^{2}+1\right)^{2}} \frac{d x}{d T}-\frac{2 x^{2}+5}{x^{2}+1}\right] .
$$


On the other hand, dividing the hydrostatic equation

$$
\frac{d P}{d r}=\frac{8 A x^{4}}{\left(x^{2}+1\right)^{1 / 2}} \frac{d x}{d r}=-\frac{G m(r)}{r^{2}} \rho
$$

by equation (23), we get

$$
\frac{d x}{d T}=\frac{2 \pi a c G}{3 A} \frac{M}{L} \frac{T^{3}}{\kappa} \frac{\left(x^{2}+1\right)^{1 / 2}}{x^{4}} \frac{m(r) / M}{L(r) / L},
$$

where $L(r)$ is the total flux $4 \pi r^{2} F$ across the sphere of radius $r, L$ is the luminosity of the star, and $M$ its total mass.

The variation of $F_{C}$, according to equation (25), is

$$
\frac{\delta F_{C}}{F_{C}}=\frac{\delta F_{R}}{F_{R}}-4 \frac{\delta T}{T}+2 \frac{\delta \rho}{\rho}\left[1+\frac{1-Q}{3 Q \ln Q}\right],
$$

which, on introduction of the values (19), (20), and (27), reduces to

$$
\frac{\delta F_{C}}{F_{C}}=2 \xi\left[\frac{T x}{\left(x^{2}+1\right)^{2}} \frac{d x}{d T}-\frac{3 x^{2}+4}{x^{2}+1}-\frac{1-Q}{Q \ln Q}\right] \text {. }
$$

Combining equations (27) and (29), we finally get, for the variation of the total flux, $\delta\left(4 \pi r^{2} F\right)=8 \pi r^{2} F \xi\left[1+\frac{T x}{\left(x^{2}+1\right)^{2}} \frac{d x}{d T}-\frac{\left(3 x^{2}+4\right)\left(\kappa_{R} / \kappa_{C}\right)+2 x^{2}+5}{\left(1+\kappa_{R} / \kappa_{C}\right)\left(x^{2}+1\right)}\right.$

where $d x / d T$ has the value given in equation (28).

$$
\left.+\frac{(Q-1)\left(\kappa_{R} / \kappa_{C}\right)}{Q \ln Q\left(1+\kappa_{R} / \kappa_{C}\right)}\right] \text {, }
$$

\section{b) GASEOUS ENVELOPE}

Here we shall adopt for the radiative opacity the general form of Kramer's law,

$$
\kappa_{R}=\kappa_{0} \rho^{n} T^{-8} ;
$$

and, for the sake of generality, we shall still take into account the possible influence of conduction, again using Kothari's expression for $F_{c}$ in nondegenerate matter,

where

$$
F_{C}=\beta^{\prime} F_{R} \frac{\rho^{2} T^{-4}}{\ln S} \quad \text { or } \quad \kappa_{C}=\frac{\kappa_{R}}{\beta^{\prime} \rho^{2}} T^{4} \ln S,
$$

$$
S=1+\vartheta^{\prime} T^{2} \rho^{-2 / 3}
$$

Proceeding as before and taking $\Gamma_{T}=\gamma=\frac{5}{3}$ in equation (20), we have

$$
\delta F_{R}=2 \xi F_{R}\left[1+\frac{3}{2} n-(s+4)\right]
$$

and, after evaluating $\delta F_{C}$ from equation (32), we finally obtain

$$
\delta\left(4 \pi r^{2} F\right)=8 \pi r^{2} F \xi\left[2+\frac{3}{2} n-(s+4)+\frac{\left(\kappa_{R} / \kappa_{C}\right)[1+(S-1) / S \ln S]}{1+\kappa_{R} / \kappa_{C}}\right] .
$$

\section{APPLICATIONS}

As we mentioned in the introduction, the question of the generation of energy in white dwarfs raises many difficulties. If thermonuclear reactions are adopted, the two following 
cases have been considered. In the first one, the abundances of the elements, although particular, are uniform through the star, and, owing to the greater densities, the reactions take place mainly in the internal part of the degenerate core. ${ }^{5}$ Depending on the presence or absence of carbon and nitrogen, the determining process will be the carbon cycle or the proton-proton reaction, although for the latter some uncertainty exists.

In the other case ${ }^{12}$ the core is practically devoid of hydrogen, which is supposed to be concentrated in the gaseous envelope. The transition region is very narrow, and in it hydrogen and heavy elements occur simultaneously in rapidly varying proportions. This zone can be the seat of nuclear reactions such as the carbon cycle. If the abundances of $C$ and $N$ were everywhere extremely low, the proton-proton reaction could still be of interest in the transition region and at the base of the gaseous envelope. It is not our purpose here to discuss the origin of such a stratification, but, although there are serious difficulties to account for it either by gravitational separation or evolutionary exhaustion, it has, however, the advantage, from the point of view of instantaneous hydrostatic equilibrium, of introducing some quantitative agreement without resorting to anomalous abundances. These two cases will be discussed separately.

a) GENERATION OF ENERGY IN THE DEGENERATE CORE

The part of the integral in condition (18) depending on the generation of energy in which we introduce equations (19) and (21) reduces to

$$
(3 \xi)^{2}\left[\overline{\left(\Gamma_{T}-1\right)}+\nu \overline{\left(\Gamma_{T}-1\right)^{2}}\right] \int_{0}^{M} \epsilon_{0} d m=(3 \xi)^{2}\left[\overline{\left(\Gamma_{T}-1\right)}+\nu \overline{\left(\Gamma_{T}-1\right)^{2}}\right] L,(36)
$$

where the mean values are computed with respect to $\epsilon$,

$$
\left.\overline{\left(\Gamma_{T}-1\right)}=\int_{0,}^{M} d \Gamma_{T}-1\right) \epsilon d m, \quad \text { etc. }
$$

On the other hand, integrating the second term in condition (18) by parts and taking into account the boundary conditions at the center, we obtain

$$
\begin{aligned}
\int_{0}^{M}\left(\Gamma_{T}-1\right) \frac{d \delta\left(4 \pi r^{2} F\right)}{d m} \frac{\delta \rho}{\rho} d m=-\frac{2}{3}\left[\delta\left(4 \pi r^{2} F\right)\right. & 3 \xi]_{M} \\
& +3 \xi \int_{0}^{M} \delta\left(4 \pi r^{2} F\right) d\left(\Gamma_{T}-1\right),
\end{aligned}
$$

or, since $\Gamma_{T}$ is a constant in the gaseous envelope,

$$
\begin{aligned}
\int_{0}^{M}\left(\Gamma_{T}-1\right) \frac{d \delta\left(4 \pi r^{2} F\right)}{d m} \frac{\delta \rho}{\rho} d m=-\frac{2}{3}\left[\delta\left(4 \pi r^{2} F\right) 3 \xi\right]_{M} & \\
& -3 \xi \int_{0}^{M_{d}} \delta\left(4 \pi r^{2} F\right) \frac{2 x d x}{3\left(x^{2}+1\right)^{2}} .
\end{aligned}
$$

We must now introduce the appropriate values for $\delta\left(4 \pi r^{2} F\right)$ in equation (38), i.e., expression (35), where $\left(\kappa_{R} / \kappa_{C}\right)$ is negligible in the integrated part, and expression (30), where $\left(\kappa_{R} / \kappa_{C}\right)$ and $Q$ can be considered as very large compared to 1 , in the last integral. Then, grouping expressions (36) and (38), the condition of vibrational stability for this case can be written

$$
\begin{aligned}
9 \xi^{2} L\left\{\overline{\left(\Gamma_{T}-1\right)}+\nu \overline{\left(\Gamma_{T}-1\right)^{2}}+\frac{2}{9}[4+3 n-2(s+4)]\right. \\
\left.\quad+\frac{4}{3} \int_{0}^{M_{d}} \frac{L(r)}{L} \frac{x d x}{3\left(x^{2}+1\right)^{2}}\left[\frac{T x}{\left(x^{2}+1\right)^{2}} \frac{d x}{d T}-2-\frac{1}{x^{2}+1}+\frac{1}{\ln Q}\right]\right\} \leqslant 0 .
\end{aligned}
$$

${ }^{12}$ C. L. Critchfield, Ap. J., 96, 1, 1942; E. Schatzman, Ann. d'ap., 8, 143, 1945. 
b) GENERATION OF ENERGY AT THE INTERFACE BETWEEN THE DEGENERATE CORE AND THE GASEOUS ENVELOPE

We will suppose that the generation of energy takes place in a thin layer at the base of the gaseous envelope. In that case the contribution of the generation of energy to condition (18) reduces to

$$
\int_{M_{d}}^{M} \frac{2}{3} \delta \epsilon \frac{\delta \rho}{\rho} d m=9 \xi^{2} \frac{2}{3}\left[1+\frac{2}{3} \nu\right] L .
$$

In equilibrium the degenerate core should be isothermal, since there is no generation of energy in it, and the equilibrium flux $F$ in that region should be zero. During the pulsation there will appear a gradient of temperature equal to

$$
\frac{d(T+\delta T)}{d m}=\frac{d \delta T}{d m}=-3 \xi T \frac{d \Gamma_{T}}{d m}
$$

and, according to equation (23), the corresponding flux $\delta F$ will be given by

$$
\delta F=\frac{16 \pi r^{2} c}{\kappa} a T^{4} \xi \frac{d \Gamma_{T}}{d m}
$$

or, since $F$ is equal to zero,

$$
\delta\left(4 \pi r^{2} F\right)=\frac{16 \pi r^{2} c a T^{4} \xi}{\kappa \rho} \frac{d \Gamma}{d r} .
$$

One can easily prove that one recovers the same expression from the general equation (30) by keeping only the term containing the indeterminate product $F(d x / d T)$ and using equations (28) and (29) and the definition of $\Gamma_{T}$.

In the gaseous envelope, $\delta\left(4 \pi r^{2} F\right)$ is still given by equation (35). Integrating by parts the term depending on the flux in condition (18), we again obtain equation (38), since it can be shown that $\delta\left(4 \pi r^{2} F\right)$ is still continuous across the boundary of the degenerate core.

But now we must introduce expression (43) for $\delta\left(4 \pi r^{2} F\right)$ in the integral of equation (38), while the integrated part reduces to the same expressions as before, so that the condition of vibrational stability becomes

$$
\xi^{2} L\left[6 n-4 s-2+4 \nu-\int_{0}^{M} \frac{48 \pi^{2} c a}{L} \frac{T^{4} r^{2}}{\kappa \rho}\left(\frac{d \Gamma_{T}}{d r}\right)^{2} d r\right] \leqslant 0 .
$$

The last term contributes to the stability of the star, but we shall see that it is practically negligible. The position of the generation of energy is not very critical either, provided that it is near enough to the surface so that the effective ratio of specific heats is close to its value $\frac{5}{3}$.

\section{NUMERICAL RESULTS}

The application of these formulae requires the use of a model, and we took for that purpose the model $\left(M=1.95 \times 10^{33} \mathrm{gm}, L=9.72 \times 10^{31} \mathrm{erg} / \mathrm{sec}, R=5.7 \times 10^{8} \mathrm{~cm}\right)$ computed by Marshak. ${ }^{5}$ The corresponding values of $\Gamma_{T}$ and some other of the expressions appearing in conditions (39) and (44) are collected in Table 1 . The numerical value of $\vartheta$ in $Q$ (eq. [26]) was taken equal to $3 \cdot 10^{-3}$, and $\kappa$ in condition (44) reduces in practice to $\kappa_{C}$ as given by equation $(24)$, where $(a / \beta)=3.5 \times 10^{-6}$.

The numerical value of the integral in condition (39) turns out to be -0.17 , while the integral in condition (44), taken with its sign, is of the order of -0.03 . This shows that these integrals play only a secondary role as compared to the other terms; this is not 
peculiar to this model but would remain true for all white dwarfs, because these integrals arise only from the rate of variation of $\Gamma_{T}$ in the star, which is slight in any case.

Taking into account the fact that, for the distribution of temperature found by Marshak, $\epsilon$ varies in the star practically as $\rho$, the mean values of $\left(\Gamma_{T}-1\right)$ and $\left(\Gamma_{T}-1\right)^{2}$ are

$$
\overline{\Gamma_{T}-1}=0.41 \quad \text { and } \quad \overline{\left(\Gamma_{T}-1\right)^{2}}=0.168 .
$$

With these values and adopting for $n$ and $s$ the values corresponding to Kramer's law, $n=1, s=3.5$, conditions (39) and (44) give the following critical values, $\nu_{c}$, of $\nu$ :

a) Generation of energy in the core:

$$
\nu \leqslant\left(\nu_{c}\right)_{1}=9.5 \text {. }
$$

TABLE 1

\begin{tabular}{c|c|c|c|c|c|c}
\hline \hline$m(r) / M$ & $x$ & $\rho / \rho_{c}$ & $T \times 10^{-6}$ & $\Gamma_{T}$ & $2 x / 3\left(x^{2}+1\right)^{2}$ & $L(r) / L$ \\
\hline 0.000 & 2.425 & 1.000 & 15.20 & 1.3818 & 0.03416 & 0.000 \\
.0045 & 2.409 & 0.980 & 15.20 & 1.3823 & .03471 & 0.003 \\
.0214 & 2.367 & 0.930 & 15.20 & 1.3838 & .03620 & 0.022 \\
.0562 & 2.297 & 0.850 & 15.20 & 1.3861 & .03888 & 0.069 \\
.111 & 2.205 & 0.752 & 15.20 & 1.3902 & .04278 & 0.138 \\
.184 & 2.094 & 0.644 & 15.18 & 1.3952 & .04816 & 0.228 \\
.272 & 1.971 & 0.537 & 15.16 & 1.4016 & .05507 & 0.344 \\
.370 & 1.839 & 0.436 & 15.14 & 1.4094 & .06387 & 0.470 \\
.471 & 1.702 & 0.346 & 15.11 & 1.4188 & .07466 & 0.587 \\
.569 & 1.563 & 0.268 & 15.07 & 1.4302 & .08787 & 0.690 \\
.662 & 1.425 & 0.203 & 15.03 & 1.4438 & .1034 & 0.782 \\
.745 & 1.291 & 0.151 & 14.98 & 1.4583 & .1210 & 0.855 \\
.817 & 1.158 & 0.109 & 14.91 & 1.4761 & .1408 & 0.920 \\
.875 & 1.029 & 0.0765 & 14.83 & 1.4954 & .1618 & 0.958 \\
.922 & 0.9039 & 0.0518 & 14.73 & 1.5168 & .1825 & 0.980 \\
.956 & 0.7801 & 0.0333 & 14.60 & 1.5408 & .2010 & 0.986 \\
.979 & 0.6571 & 0.0199 & 14.45 & 1.5662 & .2137 & 0.990 \\
.992 & 0.5293 & 0.0104 & 14.10 & 1.5937 & .2158 & 1.000 \\
0.999 & 0.3940 & 0.09429 & 13.60 & 1.6196 & 0.1982 & 1.000 \\
\hline
\end{tabular}

b) Generation of energy in a shell close to the surface:

$$
\nu \leqslant\left(\nu_{c}\right)_{2}=2.6 \text {. }
$$

As the values $\overline{\Gamma_{T}-1}$ and $\overline{\left(\Gamma_{T}-1\right)^{2}}$ depend on the values reached by $x$ toward the center of the star, they could vary with the mass of the star considered, and the value of $\left(\nu_{c}\right)_{1}$ would be a little smaller for smaller masses ( $x$ smaller at the center) and a little larger for larger masses ( $x$ larger at the center). The maximum value of $\left(\nu_{c}\right)_{1}$ is about 13 and corresponds to a configuration very close to the critical mass of Chandrasekhar. Otherwise, the integrals in conditions (39) and (44) being small, these critical values of $\nu_{c}$ will remain good approximations for practically all white dwar $s$.

\section{THE EFFECTIVE VALUE $\nu_{e}$ OF THE EXPONENT OF THE TEMPERATURE IN THE LAW OF GENERATION OF ENERGY IN A PULSATING WHITE DWARF}

If we consider a chain of nuclear reactions comprising $n$ combinations and $m$ disintegrations, the generation of energy per unit mass can be written

$$
\epsilon=\frac{1}{\bar{m}}\left(\sum_{i=1}^{n} K_{i} x_{p} x_{q} N \epsilon_{i}+\sum_{j=1}^{m} \lambda_{j} x_{j} \epsilon_{j}\right)
$$


where $N$ is the total number of particles per unit volume and $\bar{m}$ is the average mass of a particle; $x_{p}$ and $x_{q}$ are the abundances by number of particles of kinds $p$ and $q$ involved in the $i$ reaction and $K_{i} x_{p} x_{q} N^{2}$ is the number of these reactions per unit volume and unit time. The probability of disintegration of the $j$ element is $\lambda_{j}$, and $\epsilon_{i}$ and $\epsilon_{j}$ are the corresponding energies liberated. The $K_{i}$ 's depend only on temperature, and during a pulsation they will vary according to the law

$$
K_{i}=K_{i}^{0}\left(1+\nu_{i} \frac{\delta T}{T} e^{i \sigma t}\right),
$$

while the $\lambda_{j}$ 's are independent of temperature and remain constant. The variations of $N$ can, with a very good approximation, be represented by

$$
N=N_{0}\left(1+\frac{\delta \rho}{\rho} e^{i \sigma t}\right)
$$

Eddington pointed out that, if the abundances $x_{i}$ do not remain in phase with the pulsation, this could introduce delays in the generation of energy which might affect considerably the stability of the star. S. Rosseland and G. Randers ${ }^{13}$ starting from the equations of continuity for each element involved in the chain and equation (49), studied the form of the solutions for the $x_{i}$ 's and stressed in particular the importance for this question of the ratio of the mean lives of the disintegrating elements to the period of pulsation.

Bethe ${ }^{14}$ showed that, in the case of the carbon cycle, these effects would be negligible for ordinary stars, while in the case of the proton-proton reaction they would decrease or increase considerably the value of $\nu$ with respect to its hydrostatic value, according to whether the period of pulsation is smaller or larger than the mean life of $B e^{7}$.

For the model of Sirius B treated here, a period $P$ of the order of 10 seconds was found in Paper I, and in a first approximation we can find the period for any other model by using the relation $P \sqrt{ } \bar{\rho}=$ Constant where $\bar{\rho}$ is the mean density.

\section{a) THE CARBON CYCLE}

In this case, there are two radioactive disintegrations,

$$
N^{13} \rightarrow C^{13}+\epsilon^{+} \quad \text { and } \quad O^{15} \rightarrow N^{15}+\epsilon^{+},
$$

the mean lives of $N^{13}$ and $O^{15}$ being, respectively, of the order of 10 and 2 minutes. The mean life of the other elements can be obtained by multiplying the values given by Bethe $^{15}$ for the sun by a factor $\left(\rho x_{H} \tau^{2} e^{-\tau}\right)_{\odot} /\left(\rho x_{H} \tau^{2} e^{-\tau}\right)$, where $x_{H}$ is the abundance of hydrogen and $\tau$ is the quantity defined by Bethe and is inversely proportional to $T^{1 / 3}$. As the generation of energy per unit mass is directly proportional to $\rho x_{H} \tau^{2} e^{-\tau}$ and since it must be much less in a white dwarf than in the sun, one has to take abundances of hydrogen small enough to render the ratio $\left.\left(\rho x_{H} \tau^{2} e^{-\tau}\right)_{\odot} / \rho x_{H} \tau^{2} e^{-\tau}\right)$ much greater than 1 . Hence the mean lives of these elements will be longer in a white dwarf than in the sun.

Even if the reactions take place in a shallow layer near the surface, the mean lives of these elements will still be at least as long as in the sun, so that in all cases the abundances of $C^{12}, C^{13}, N^{14}$ and $N^{15}$ can be treated as constant during periods of time long compared to the periods of pulsations.

As far as the two disintegrations are concerned, the energy liberated is so small that

${ }^{13}$ Astrophysica Norvegica, 3, 71, 1933; cf. also S. Rosseland, op. cit., chap. v, §§ 3, 4, 5.

${ }_{14}$ Phys. Rev., 55, 434, 1939.

${ }^{15}$ For corrections to the original values cf. H. A. Bethe, Ap. J., 92, 118, 1940, and W. A. Fowler and R. N. Hall, Phys. Rev., 74, 1558, 1948. 
it does not matter much whether it is delayed or not with respect to the pulsation, and for all practical purposes we can neglect the second term in the right-hand member of equation (47). Denoting the rate of generation of energy in equilibrium by $\epsilon_{0}$, this equation can then be written

$\epsilon=\epsilon_{0}\left[1+\left(\frac{\epsilon_{1}+\epsilon_{3}+\epsilon_{4}+\epsilon_{6}}{\epsilon_{1}+\epsilon_{2}+\epsilon_{3}+\epsilon_{4}+\epsilon_{5}+\epsilon_{6}}\right) \frac{\delta \rho}{\rho} e^{i \sigma t}+\left(\frac{\epsilon_{1} \nu_{1}+\epsilon_{3} \nu_{3}+\epsilon_{4} \nu_{4}+\epsilon_{6} \nu_{6}}{\epsilon_{1}+\epsilon_{2}+\epsilon_{3}+\epsilon_{4}+\epsilon_{5}+\epsilon_{6}}\right) \frac{\delta T}{T} e^{i \sigma t}\right]$

which is strictly correct when the period of pulsation is shorter than the mean lifetime of $N^{13}$ and $O^{15}$, which is the case in white dwarfs. As we have already said, the energies $\epsilon_{2}$ and $\epsilon_{5}$ liberated by the disintegrations are so small that we can put the coefficient of $\delta \rho / \rho$ equal to 1 .

Taking into account the variations of $\tau$ and $\nu$ with the range of temperatures to which the formula must apply and using the integrations of Marshak, ${ }^{5}$ one finds for $\nu_{e}$, which is the coefficient of $\delta T / T$ in equation (50), the following values: for Sirius B, generation of energy in the central region, $\nu_{e}=19$, and generation of energy in an external shell, $\nu_{e}=22$; for 40 Eridani B, generation of energy in the central region, $\nu_{e}=15$, and generation of energy in an external shell, $\nu_{e}=22$.

The temperatures obtained by Marshak are undoubtedly too high, since they correspond to a negligible helium content, while one would normally expect a fairly high abundance of helium in a star having very little hydrogen left. Increasing the abundance of helium would decrease the temperature, and this, in turn, would again increase the values of $\nu_{e}$. It does not seem possible to imagine cases where $\nu_{e}$ could be smaller than, say, the value given for the central region of 40 Eridani $B$.

\section{b) THE PROTON-PROTON REACTION}

In this case, since the mean life $\Lambda\left(L i^{7}\right)$ of $L i^{7}$ tends to be very short, the mean life $\Lambda\left(B e^{7}\right)$ of $B e^{7}$, from which $L i^{7}$ results by electron capture, plays a determining role in the evaluation of $\nu_{e}$.

The probability of capture of an electron by $B e^{7}$ in a white dwarf is proportional in a first approximation to

$$
N W^{2}\left[1+\frac{g(x)}{4 W x^{3}}+\frac{1}{W^{2}}\left\{\left[\left(1+x^{2}\right)^{1} /^{2}-1\right]^{2}-\frac{2}{5} x^{2}+\frac{f(x)}{4 x^{3}}\right\}\right]
$$

where $x=p_{0} / m c$ has the same meaning as before and $g(x)$ and $f(x)$ are the functions defined by equations (5) and (7) and tabulated by Chandrasekhar on page 361 of his book. The energy $W$ is measured in units of $m c^{2}, m$ being the rest-mass of the electron, and is equal to

$$
W=\frac{1}{m}\left[m\left(B e^{7}\right)-m\left(L i^{7}\right)\right]+1
$$

where $m\left(B e^{7}\right)$ and $m\left(L i^{7}\right)$ are the masses of the corresponding nuclei. The factor multiplying $N W^{2}$ in equation (51) can be taken equal to 1 in the external part of a white dwarf, where $x$ is small; and for $x=2,5$, and 10 it takes, respectively, the values $2.3,7$, and 24 . The ratio of the number of electrons, $N$, in a white dwarf poor in hydrogen to the number of electrons in the sun is given by

$$
\frac{N}{N \odot}=\frac{\rho}{\rho \odot\left(1+x_{H}\right) \odot} .
$$

As Bethe gives 14 months for $\Lambda\left(B e^{7}\right)$ at the center of the sun, one easily computes from equations (51) and (52) that its value at the center of Sirius $B$ is $\Lambda\left(B e^{7}\right) \simeq 65$ 
seconds. In the transition region between the degenerate core and the gaseous envelope $\Lambda\left(B e^{7}\right) \simeq 2$ days.

On the other hand, again using the model computed by Marshak, one finds $\Lambda\left(L i^{7}\right)=$ 60 seconds, just as at the center of the sun, the corresponding quantity $\left(\rho x_{H} \tau^{2} e^{-\tau}\right)$ being about the same in both cases. If the generation of energy takes place in an external shell with a high abundance of hydrogen, one finds $\Lambda\left(L i^{7}\right) \simeq 90$ seconds.

The period of pulsation of Sirius B being of the order of 10 seconds, one finds by integrating the equations of continuity that the abundances $x_{1}, x_{3}, x_{4}, x_{5}$ of $H, H e^{3}, B e^{7}$, and $L i^{7}$ can again be considered as constant and equal to their original values, while the abundance $x_{2}$ of $H^{2}$, which has an extremely short life-smaller than 1 second-is given by

$$
x_{2}=\frac{K_{1}^{0} x_{1}}{K_{2}^{0}}\left[1+\left(\nu_{1}-\nu_{2}\right) \frac{\delta T}{T} e^{i \sigma t}\right] .
$$

Introducing these abundances into the corresponding equation (47), one gets

$$
\boldsymbol{\epsilon}=\epsilon_{0}\left[1+\left(\frac{\delta \rho}{\rho}\right) e^{i \sigma t}+\frac{\left(\epsilon_{1}+\epsilon_{2}\right) \nu_{1}+\epsilon_{3} \nu_{3}+\epsilon_{5} \nu_{5}}{\left(\epsilon_{1}+\epsilon_{2}+\epsilon_{3}+\epsilon_{4}+\epsilon_{5}\right)} \frac{\delta T}{T} e^{i \sigma t}\right]
$$

Using the appropriate values in the coefficient of $(\delta T / T)$, one finds, for the center of Sirius B,

$$
\nu_{e}=10.7
$$

The case of the external layer is similar, since the $\Lambda^{\prime}$ s, except $\Lambda_{2}$, are still all larger than the period of pulsation.

In the case of 40 Eridani $B$, if we take for the temperature the values computed by Marshak, we find

$$
\Lambda\left(B e^{7}\right) \simeq 20 \text { minutes }, \quad \Lambda\left(L i^{7}\right) \simeq \frac{1}{2} \text { second },
$$

while the period of pulsation is approximately equal to 40 seconds. Thus $\Lambda\left(L i^{7}\right)$ is now smaller than the period, and the abundance $x_{5}$ of $L i^{7}$ can no longer be treated as a constant but is given in a first approximation by

$$
x_{5}=x_{5}^{0}\left[1-\nu_{5} \frac{\delta T}{T} e^{i \sigma t}\left(1-\frac{2 \pi i \Lambda L i^{7}}{P}\right)\right] .
$$

Introducing expressions (53) and (55) into the corresponding equation (47) and treating the other abundances as constant, we obtain in this case

$$
\epsilon=\epsilon_{0}\left\{1+\frac{\delta \rho}{\rho} e^{i \sigma t}+\frac{\delta T}{T} e^{i \sigma t}\left[\left(\epsilon_{1}+\epsilon_{2}\right) \nu_{1}+\epsilon_{3} \nu_{3}+\epsilon_{5} \nu_{5} \frac{2 \pi i \Lambda L i^{7}}{P}\right]\right\} .
$$

The last term in the coefficient of $\delta T / T$ can be neglected in any case, since it is out of phase by $90^{\circ}$ and cancels out in the course of a period. The value of $\nu_{e}$ is now much smaller and equal to 1.8 .

In the external regions of 40 Eridani $\mathrm{B}, \Lambda\left(\mathrm{Li}^{7}\right)$ becomes much larger and of the order of 120 seconds, i.e., larger than the period of pulsation, and $\epsilon$ is again given by formula (54), $\nu_{e}$ again being of the order of 10-11.

We must notice, however, that, as we mentioned before, the temperatures given by Marshak are probably too high. A large abundance of helium would reduce the central 
temperature of 40 Eridani B by a factor of the order of $\frac{1}{2}$, and this would bring back $\Lambda\left(L i^{7}\right)$ to a value greater than the period of pulsation and $\nu_{e}$ to a value of the order of 10-11. In Sirius B a large abundance of helium would also increase slightly the value of $\nu_{e}$.

VII. CONCLUSIONS

We have collected the main results of the preceding discussion in Table 2. A com-

TABLE 2

VALUES OF $\nu_{e}$ AND $\nu_{c}$

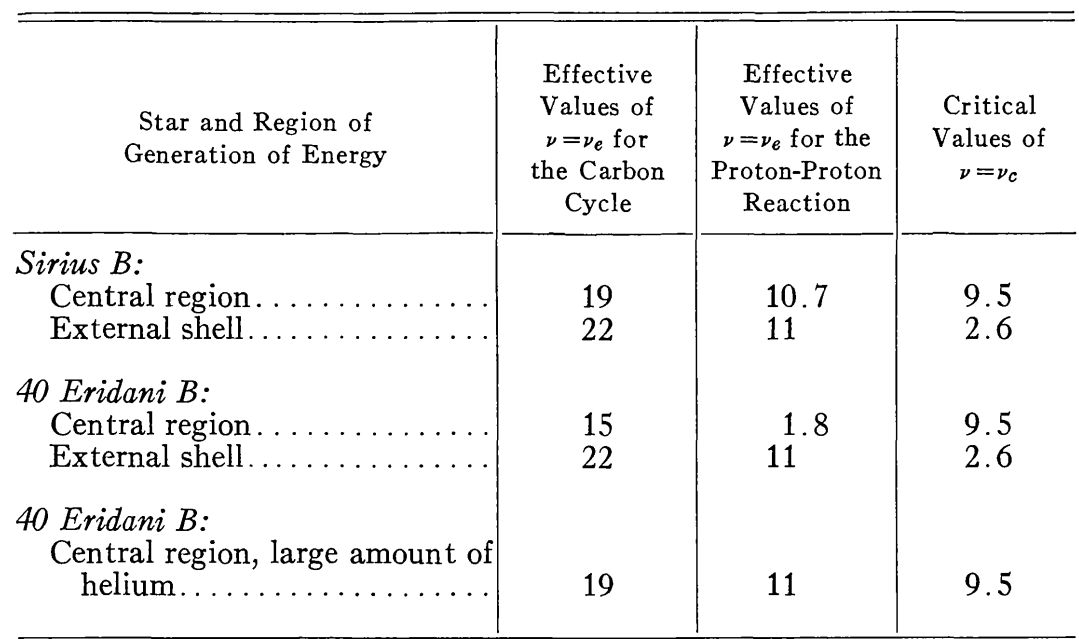

parison between the values of $\nu_{e}$ for the carbon cycle and the critical values $\nu_{c}$ shows immediately that this reaction cannot take place inside a white dwarf either at the center or in the external layers. The same applies for the proton-proton reaction, as far as the external layers are concerned. In the interior of Sirius B the value of $\nu_{e}$ and $\nu_{c}$ for this reaction are very close, and our computations are perhaps not precise enough to be absolutely sure of the sign of $\left(\nu_{e}-\nu_{c}\right)$. However, in any case, the margin of stability would be very small.

In 40 Eridani B we see that, if the helium content is negligible, the proton-proton reaction could, in fact, take place toward the center without endangering the stability. However, it seems likely that the helium abundance will be large, and, as we saw before, in that case the value of $\nu_{e}$ becomes again of the order of 10-11.

In the preceding discussion, when we referred to the proton-proton reaction, we always assumed that the Gamow-Teller selection rules were applicable. If one reverted to the primitive Fermi rules, the reaction would become less probable by a factor of the order of $10^{6}$. In that case and if $C$ and $N$ were absent from the star, the necessary abundance of $H$ to explain the generation of energy would turn out to be much larger and of the same order as in ordinary stars. But this, in turn, would reduce considerably the central temperature and also the density, so that the mean life of $\mathrm{Li}^{7}$ would again become larger in all cases than the period of pulsation, and the corresponding values of $\nu_{e}$ would tend again to values of the order of 10-12, bringing about vibrational instability. The critical values of $\nu_{c}$ will change very little from one star to another, as the variable terms in equations (39) and (44) are very small.

One can also remark that the period of pulsation decreases as $(\bar{\rho})^{-1 / 2}$, while the mean life of $B e^{7}$ decreases as $(\bar{\rho})^{-1}$; and thus, if we go to white dwarfs of larger and larger densities, the mean life of $\mathrm{Be}^{7}$ soon becomes smaller than the period of pulsation, and in that 
case the value of $\nu_{e}$ for the proton-proton reaction is at least of the order of 11 or 12 in all cases. For instance, in the case of the white dwarf (AC $70^{\circ} 8247$ ), taking the value of $\bar{\rho}$ given by $\mathrm{S}$. Chandrasekhar, ${ }^{16}$ one finds that $\Lambda\left(B e^{7}\right)$ is reduced to 1.5 seconds, while the period of pulsation is 3 seconds. Thus, altogether, it appears extremely unlikely that nuclear reactions could be the source of the energy radiated by white dwarfs.

${ }^{16}$ Op. cit., p. 431. 